\title{
In vitro model for the study of necrosis and apoptosis in native cartilage
}

\author{
Shawn Patrick Grogan,' Balz Aklin,' Martin Frenz,' Thomas Brunner,' Thomas Schaffner' \\ and Pierre Mainil-Varlet ${ }^{\mid *}$ \\ I Institute of Pathology, University of Bern, Bern, Switzerland \\ 2 Institute of Applied Physics, University of Bern, Bern, Switzerland
}

*Correspondence to:

Dr Pierre Mainil-Varlet, Institute of Pathology, University of Bern, Murtenstrasse 31, Bern 3010, Switzerland.

E-mail:mainil@patho.unibe.ch

\begin{abstract}
Apoptosis plays a role in everything from early development to ageing and in a host of disease states. Studying this important process in the in vivo state is critical, to understand its varied role and to open further avenues of therapeutic intervention. The present paper presents an ex vivo bovine articular cartilage model to study apoptotic and necrotic processes following acute injury. Ex vivo bovine articular cartilage was assessed 1, 3 and 6 days following holmium : YAG laser treatment $(780 \mathrm{~mJ})$. Markers to visualize cell viability, caspase-3 activity, changes in mitochondrial membrane potential and the degree of DNA fragmentation (TUNEL assay) were used alone or in various combinations. Standard histology and transmission electron microscopy (TEM) were also performed for a more comprehensive assessment. A significant progression $(p<0.05)$ of ethidium/caspase-3-positive signal depth at day 3 preceded a significant increase $(p<0.05)$ in TUNEL signal depth by day 6 . The mitochondrial matrix marker CMXRos was shown to provide an alternative to calceinAM for assessing cell viability. The identification of chondrocyte apoptosis morphology by TEM was not conclusive. Nevertheless, TEM revealed that cells which were clearly necrotic also stained positively for TUNEL, thus indicating the risk of using TUNEL alone for the assessment of apoptosis. The model described here allows the rapid, spatial and temporal determination of cell viability and of apoptotic and necrotic processes in whole-tissue specimens after acute injury, and permits study of the balance between these events. The assessment of healthy and diseased cartilage and of the effects of surgical, pharmaceutical or in vitro intervention are immediate applications of these protocols. Moreover, this model may be useful for the study of key mechanisms involved in apoptosis or for the establishment of other markers of apoptosis.
\end{abstract}

Keywords: apoptosis; confocal microscopy; cartilage; caspase-3; TUNEL; ethidium homodimer

\section{Introduction}

Apoptosis is a important component of normal development, growth, immunoregulation and homeostasis. It is also involved in the pathophysiology of many clinical disorders, including myocardial ischaemia, stroke, neurodegenerative disease, pulmonary inflammatory disease, inflammation, myelodysplastic disorders, organ transplantation and oncology [1-3]. Tissue viability following surgical intervention or tissue engineering is critical for the long-term success of many procedures; understanding the responses of various tissues to insult may lead to improved treatments. Methods to assess cell viability have been utilized in a variety of fields including our focus area, orthopaedic research [4-6], but on its own, the assessment of viability in terms of the living or dead state immediately after an injury is perhaps not sufficient to predict longer-term tissue survival. Indeed, a number of investigators have employed methods to detect apoptosis in a range of situations, including endochondral ossification [7], diseased joints [8-11], a cartilage mechanical model [12], following impact loading [13], subsequent to matrix depletion [14] and in chondrocyte cell cultures $[15,16]$.

Cell death is usually classified into two broad categories: apoptosis and necrosis. Necrosis is a passive catabolic and pathological process that represents a cell's response to extreme accidental or toxic insult. Apoptosis, by contrast, occurs under normal physiological conditions and is an active process requiring energy. It can also be elicited by toxic injury or during a disease process. In these non-physiological conditions, both types of cell death can be encountered following the same initial insult and the balance between death by apoptosis and by necrosis appears to depend upon the intensity of injury and the level of available intracellular ATP [17].

Although the importance of apoptosis in regulating cellular homeostasis through control of cell death 
is undisputed, distinguishing an apoptotic cell from one dying, and from accidental cell death (ACD), is a laborious task [18]. Kerr et al. [19] were the first formally to describe the characteristic morphological features of apoptosis as a form of physiological cell death distinctly different from necrosis. Morphological features detectable under light microscopy, such as nuclear fragmentation, were present for a short period of time and only during the final stage of the process. Electron micrographs of apoptotic cells displayed shrinkage and nuclear chromatin condensation into dark spheres or crescents that budded into 'apoptotic bodies', which were then absorbed by the surrounding tissue, preventing inflammation. Necrosis, on the other hand, is characterized by cells that swell and decay in an unorganized manner, leading to an inflammatory response [20]. Nearly three decades following these observations, much progress has been made to identify the key components driving this organized death pathway. Central components that apparently coordinate this disassembly of the cell involve the mitochondria $[21,22]$ and a family of cysteine proteinases called caspases [23].

Various methods are available to assess such apoptotic processes in situ, but they often involve complicated procedures that can be time consuming. Previously, we have studied laser-induced damage in cartilage [24], demonstrating its excellent cutting and optical qualities, along with the ability to culture this nonmetabolically demanding tissue. Subsequently, the precise injury created by laser treatment prompted us to develop this system as a model for the study and evaluation of apoptotic markers. The aim of the present study, therefore, was to validate this model of injury as a method for the rapid assessment of cell viability and apoptosis, in terms of cell wall permeability, esterase activity, caspase-3 activity, mitochondrial transmembrane integrity and DNA fragmentation, over time. We adapted and combined the use of commercially available fluorescence markers to make spatial and temporal observations of these events.

\section{Material and methods}

\section{Preparation for laser treatment}

Bovine articular cartilage (AC) was derived from 3- to 6-month-old calves, 2 to 3 days after slaughter. Pieces of AC from the femoral condyle $(15 \times 15 \times 6 \mathrm{~mm})$ were aseptically separated from the subchondral bone and maintained in sterile media (DMEM F-12 HAM, Life Technologies Ltd, Paisley, UK) with 10\% FBS (Life Technologies, HyClone, UT, USA) until exposed to laser treatment $(2-3 \mathrm{~h})$. All samples were screened for viability by the Live/Dead assay (L/D assay) (Molecular Probes, Eugene, OR, USA) and fresh untreated samples with a high proportion of cell death, with an ethidium homodimer-positive signal exceeding $10-15 \%$, were not used in the study. A fibre-coupled multi-wavelength holmium:YAG laser light source was operated in a free-running mode, at a pulse repetition rate of $8 \mathrm{~Hz}$ and a pulse duration of $400 \mu \mathrm{s}$, with a $50 \%$ spot overlap between consecutive pulses. For each treatment, the laser energy was $780 \mathrm{~mJ}$, where the laser fibre tip was positioned at a $90^{\circ}$ angle to the cartilage surface at a constant distance of $1 \mathrm{~mm}$. A single line of laser exposure was applied across each treatment sample and two untreated control pieces were maintained for each time point. Subsequent to laser exposure, the pieces of cartilage $(n=12$ for each time point) were maintained in media (DMEM F-12 HAM with 10\% FBS) for 1, 3, or 6 days, before assessment by confocal microscopy or fixation for either standard histology or TEM.

\section{Preparations for analyses}

Live pieces of cartilage for confocal analyses, at each time point, were embedded in 3\% agar (aqua. Dest. Fluka, Buchs, Switzerland) and sectioned with a tissue slicer (Sorvall TC-Z Newtown, CT, USA) to produce slices $150 \mu \mathrm{m}$ thick. The cartilage slices were then incubated in a variety of buffers as indicated below. The remaining unsliced samples were divided for fixation either in 5\% glutaraldehyde for TEM, or in $4 \%$ buffered paraformaldehyde $(24 \mathrm{~h})$ for paraffin embedding and subsequent histology and standard TUNEL assessment for paraffin slices using the In Situ Death Detection Kit, TMR red (Boehringer, Roche Diagnostic, Rotkreuz, Switzerland).

\section{Live/dead assay}

Slices of cartilage (two to three) were incubated for $60 \mathrm{~min}$ in the live/dead (L/D) buffer (Molecular Probes, Eugene, OR, USA), consisting of calceinAM $(1 \mu \mathrm{M})$ and ethidium homodimer-1 $(8 \mu \mathrm{M})$ in PBS; as outlined by the manufacturer. Ethidium homodimer1 enters into cells with compromised cell membranes and subsequently intercalates with nucleic acids. Thus, a cell with compromised cell membranes has a red fluorescence and is classified as dead. Calcein-AM penetrates the membranes of living cells, where ubiquitous cytoplasmic esterases cleave the molecule to produce a green fluorescence.

\section{CasE buffer}

The CasE buffer consisted of PhiPhilux (caspase3 substrate, $8 \mu \mathrm{M}$, Oncolmmunin Inc., College Park, MD, USA) and ethidium homodimer $(8 \mu \mathrm{M})$. PhiPhilux is a peptide substrate containing the sequence GDEVDGI that is cleaved by active caspase3 at the underlined ' $D G$ ' site to produce a highly intense green fluorescence $\left(\lambda_{\mathrm{ex}}=505 \mathrm{~nm}\right.$ and $\lambda_{\mathrm{em}}=$ $530 \mathrm{~nm}$ ).

\section{CasX buffer}

PhiPhilux $(8 \mu \mathrm{M})$ and CMXRos (500 nM, Molecular Probes, Eugene, USA) were combined in this 
buffer mix to identify cells with active caspase-3 and to observe mitochondrial viability, respectively. CMXRos (Molecular Probes, Eugene, OR, USA) comprises thiol-reactive chloromethyl moieties that accumulate in intact mitochondria and are subsequently oxidized to produce a red fluorescence $\left(\lambda_{\text {ex }}=579 \mathrm{~nm}\right.$ and $\lambda_{\text {em }}=599 \mathrm{~nm}$ ). A loss of red fluorescence is indicative of a loss of mitochondrial membrane potential.

\section{TUNEL assay}

Cartilage slices were also prepared for an adapted direct TUNEL assay (In Situ Death Detection Kit, TMR red, Boehringer, Roche Diagnostic, Rotkreuz, Switzerland). Briefly, the adapted TUNEL assay consisted of washing the slices twice with PBS/1\% BSA at $4{ }^{\circ} \mathrm{C}$, fixation in $4 \%$ paraformaldehyde in PBS for $30 \mathrm{~min}$ at room temperature with intermittent mixing, washing in $\mathrm{PBS} / 1 \% \mathrm{BSA}$ twice before addition of permeabilization solution $(0.1 \%$ Triton $\mathrm{X}-100$ in $0.1 \%$ sodium citrate), and incubation on ice for $2 \mathrm{~min}$. Subsequently, cartilage slices were selected randomly for either incubation in the enzyme/label solution (test solution) or only the label solution (negative controls) at $37^{\circ} \mathrm{C}$ for $60 \mathrm{~min}$ in a dark, humid incubator. For positive controls, slices were incubated with DNAse 1 ( $1 \mathrm{mg} / \mathrm{ml}$ for $10 \mathrm{~min}$ ). Following incubation, the samples were washed twice in PBS and stored in this solution until confocal assessment within 1-2 h. All slices incubated in the above solutions were protected from direct sunlight.

\section{Histology}

Portions of the unsliced cartilage for confocal analyses were fixed in $4 \%$ paraformaldehyde in buffered PBS for $24 \mathrm{~h}$ and subsequently dehydrated and embedded in paraffin wax. Sections $5 \mu \mathrm{m}$ thick were stained with alcian blue or haematoxylin eosin.

\section{Electron microscopy}

Samples were fixed in $5 \%$ glutaraldehyde in $0.05 \mathrm{M}$ sodium cacodylate for $24 \mathrm{~h}$, post-fixed in $1 \%$ osmium tetroxide, dehydrated and embedded in Spurr's resin (EDS, Washington, PA, USA). Sections $1 \mu \mathrm{m}$ thick were stained with toluidine blue for sample assessment and orientation. Subsequently, $80 \mathrm{~nm}$ thick sections were contrasted with uranyl acetate followed by lead citrate.

\section{Methods of confocal image acquisition and assessment}

Cartilage slices that were prepared using the abovementioned fluorescence markers were visualized using a laser scanning confocal microscope (LSM410 Zeiss, Oberkochen, Germany). The boundaries of the visible live (calcein positive) and dead (ethidium positive) demarcation zone were measured with a $10 \times$ objective (using the provided LSM410 software) in terms of damage width and depth from the surface for each time point. The visible boundary of TUNEL-positive cells was measured in the same manner.

\section{Statistics}

A one-way ANOVA was used to test for differences between confocal signal measurements. Tukey's multiple comparison test was employed when significant differences $(p<0.05)$ were established with ANOVA (GraphPad Prism ${ }^{\circledR}$ Software, version 2.01, San Diego, CA, USA). $t$-Tests were employed to determine if the signal areas of ethidium and TUNEL at each corresponding time point and with respect to width and depth were significantly different. Linear regression was calculated on each variable over time.

\section{Results}

A summary of events observed for L/D, caspase3 and TUNEL signals are presented in Figure 1, which further defines specified zones to allow an overview of temporal events following laser injury. The L/D assessment clearly demarcated the zone of laser damage (Figure 2), which significantly $(p<$ 0.05 ) increased in depth by day 3 , but did not further change after 6 days (Figure 3A). Both direct LSM and standard paraffin section TUNEL assay test observations were consistent and the area of TUNEL-positive cells significantly $(p<0.05)$ increased in width and

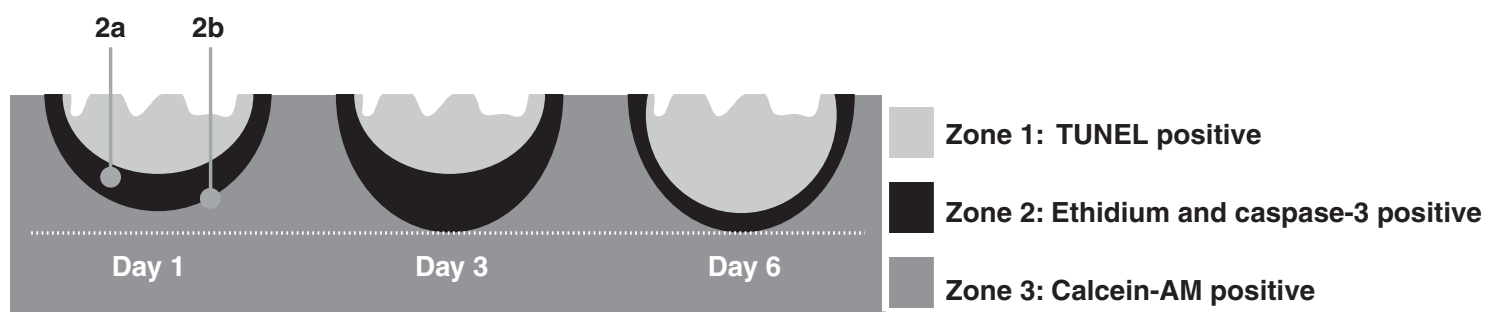

Figure I. Cartoon representing the ethidium homodimer-I, caspase-3, calcein-AM staining areas over 6 days post-holmium: YAG laser exposure. Zone $\mathrm{I}$ is designated as the TUNEL-positive zone that progresses into the ethidium/caspase-3 area (zone 2) by day 6 . Zone 2 is characterized by the co-signal of ethidium homodimer-I and active caspase-3, which progresses significantly by day 3 but does not change by day 6 . Zone $2 \mathrm{a}$ indicates a region in zone 2 that is close to the border of zone I. Zone $2 \mathrm{~b}$ is a region that is within zone 2 , but is close to the border of zone 3 . Zone 3 represents cells with a positive calcein-AM signal 

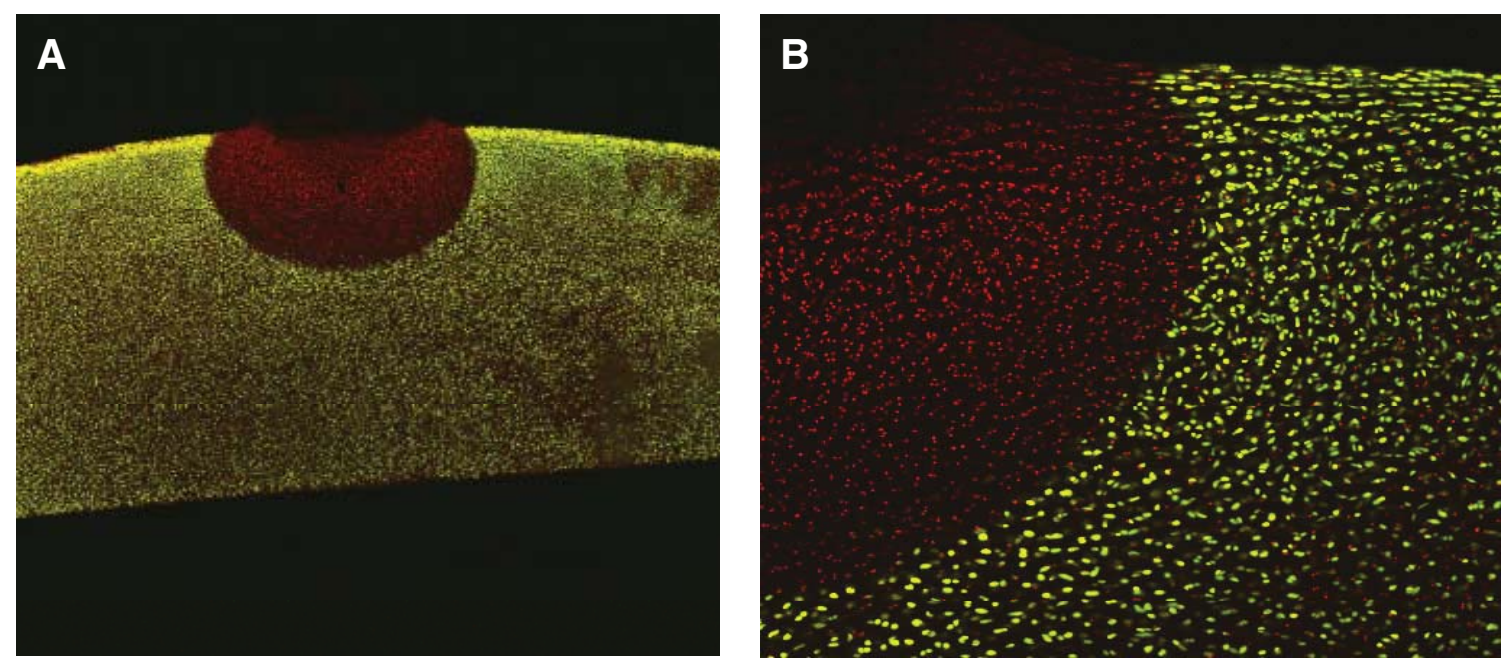

Figure 2. Bovine cartilage $24 \mathrm{~h}$ following holmium: YAG laser exposure $(780 \mathrm{~mJ})$ and incubated with the live/dead buffer. The red signal indicates cells that have compromised cell membranes and allow ethidium homodimer to enter. Calcein-AM enters into live cells with intact membranes and active cytoplasmic esterases cleave the calcein-AM substrate to produce a green fluorescence (A: $2.5 \times ; \mathrm{B}: 10 \times)$

depth over 6 days (Figures $3 \mathrm{~A}$ and 4). TUNELpositive areas were significantly smaller than those visualized by ethidium, in terms of width on day 1 $(p<0.05)$, and of both width and depth on day $3(p<$ 0.05 , Figure $3 \mathrm{~A}$ and $\mathrm{B})$. No significant difference was found when comparing ethidium and TUNEL on day 6 (Figure 3A and B). Linear regression of both ethidium and TUNEL measurements over time revealed a significant $(p<0.02)$ trend only for increased TUNEL signal over time $\left(r^{2}=0.999\right)$, although a general increase in ethidium signal area was observed. Active caspase-3 signals co-signalled in cells that were also ethidium positive, at all time points observed. Only a few randomly distributed caspase- and ethidiumpositive cells were detected beyond the sharply defined ethidium homodimer stained area (Figure 5). It was decided to use the ethidium area to co-represent caspase-3 activity, since we observed this co-signal at each time point. Although the cartilage matrix appeared to trap the PhiPhilux substrate, and produced a non-specific background signal, a bright green fluorescence produced by caspase-3-positive cells could clearly be detected. This background made accurate measurements of the caspase- 3 area alone difficult, hence the use of ethidium measurements. Mitochondrial membrane potential, assessed by the CMXRos signal, was evident in the calcein-AM (live/green) area and was not present in the ethidium/caspase-3-positive zone (Figure 6). Paraffin-embedded slices stained with alcian blue revealed matrix depletion and the formation of vacuoles at the immediate laser impact site (Figure 7). Haematoxylin-eosin and alcian blue staining showed the presence of pyknotic cells beyond the site of matrix depletion.
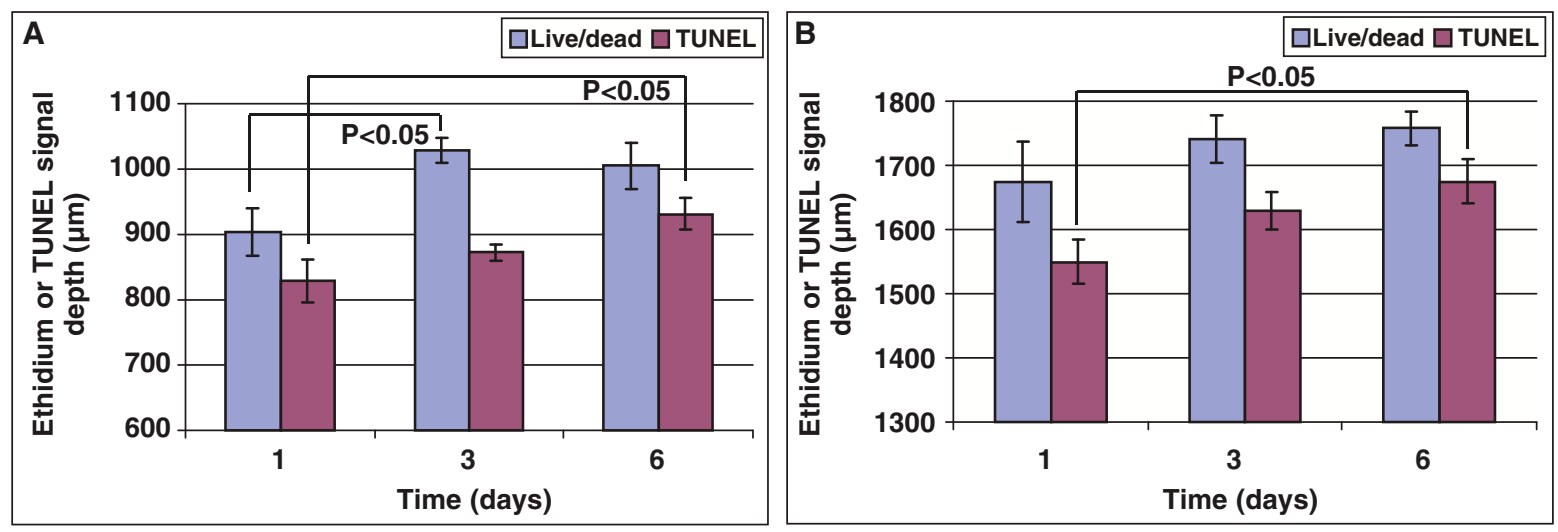

Figure 3. (A) Laser damage depth as assessed by live/dead (ethidium boundary signal) and TUNEL positive signal. A significant increase $(p<0.05)$ in the ethidium signal depth (blue bars) occurred between day I and day 3 , but did not advance significantly by day 6. TUNEL signal depth (red bars) advanced significantly $(p<0.05)$ by day 6 . The depth of TUNEL signal at days I and 3 was significantly less than the ethidium signal, but the TUNEL signal progressed deeper by day 6 and was not significantly different from the depth signal of ethidium homodimer at this time point. (B) Laser damage width as assessed by live/dead (ethidium boundary signal) and TUNEL positive signal. No significant change in the ethidium signal width (blue bars) was found between all time points studied. TUNEL signal width (red bars) advanced significantly $(p<0.05)$ by day 6 and no significant difference was found between the ethidium and TUNEL width measurements by day 6 


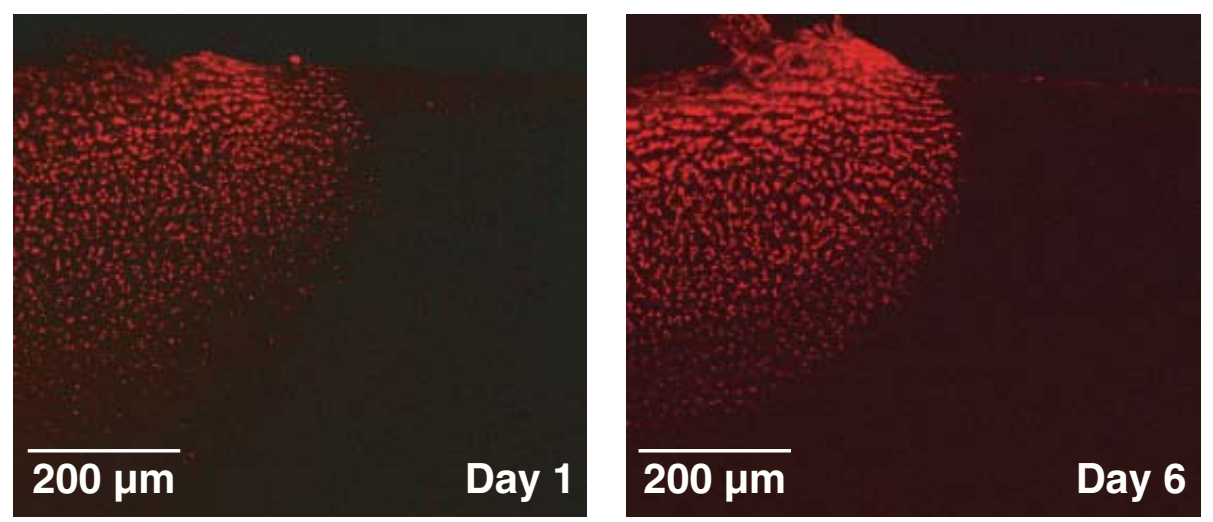

Figure 4. TUNEL signal observed at day I and day 6 following holmium: YAG laser exposure. TUNEL signal (zone I, see Figure I) progressed into zone 2 (defined by ethidium/caspase signal) by day 6. DNA fragmentation signal depth and width increased significantly $(p<0.05)$ between days I and 6 . The fragmentation signal produced on day I was predominately necrotic in nature (see Figure 8) and the progressive signal is likely to be apoptosis-derived DNA fragmentation, based on cellular morphology (Figure 8 ) and the presence of an active caspase-3 signal (Figures 5 and 6 )

At the ultrastructural level there was evidence of necrosis in zone 1 (see Figure 1), possibly due to direct laser impact. The cells in this area (zone 1) were also TUNEL positive where cellular contents were forced into the immediate surrounding matrix (Figure 8A-C). However, cells located in zone 2 (see Figure 1), located within the ethidium-positive area, showed unusual morphology and were likely to be apoptotic. The cells located in zone 2 were condensed and separated from the lacuna wall; some of their organelles were tightly packed, and cell membranes appeared intact (Figure 8E, F and H). Chondrocytes located outside the zone of ethidium-positive cells (zone 3: calcein positive) had a morphology consistent with well-preserved chondrocytes (Figure 8I).

\section{Discussion}

Following laser injury, the ethidium homodimer signal represented the best means of assessing global damage at any time period. The width of the ethidium signal did not significantly change over the 3 days and appeared to provide the best means of assessing cell/tissue viability $24 \mathrm{~h}$ after holmium laser treatment. Although the ethidium signal depth increased significantly, it provided the best way of assessing laser tissue damage, compared to standard histology or TUNEL. However, an active caspase-3 signal coexisted with the ethidium homodimer-positive signal. This co-signal was unexpected because caspase activity was predicted to precede the breakdown of the cell membrane. Nevertheless, there may be many reasons for this co-signal. The activation of caspase has been reported between $30 \mathrm{~min}$ and $3 \mathrm{~h}$ following neuronal injury $[25,26]$ and thus the damage caused by these proteolytic enzymes may have already occurred within the $24 \mathrm{~h}$ following laser treatment. Hence, we perhaps observed cells that were moving from apoptotic death to a necrotic state, with residual caspase activity. Alternatively, caspases were perhaps active beyond the ethidium border and caused damage at levels below the detection threshold of the PhiPhilux fluorescence. Another explanation for the co-staining of caspase-3 activity in ethidium-positive cells may be the occurrence of laser-induced injury, leading to a loss of membrane integrity in such a way that independently triggered caspase activation. Indeed, physical impact has been demonstrated to induce apoptosis in cartilage [12,27].

The combination of different apoptotic indicators led to the observation of temporal changes in both caspase- 3 and TUNEL signals. Within the assumption that caspase- 3 signal area equalled ethidium signal area, we observed that cells previously TUNEL negative and caspase- 3 positive at day 1 became TUNEL positive by day 6 . Although this is an expected observation [28] it clearly indicated that one might be able to track processes in situ relatively easily when employing these adapted protocols. This also illustrates that chondrocyte death following holmium laser exposure (at $780 \mathrm{~mJ}$ ) is a blended temporal mix of necrotic and apoptotic events. Comparing TUNEL and ethidium signals as predictors of laser damage, it is apparent that the TUNEL signal underestimated the total damage until day six. Thus, studies relying only on a TUNEL signal could underestimate the damage caused if visualized before the sixth day following treatment, at least in this model.

It was observed that the TUNEL signal 'caught up' with the ethidium signal area by day 6 , which implies that the progression of cell death may have ceased. Nevertheless, it was noted that caspase-3 activity was still detectable around the margins of the ethidium signal zone at day 6 , which is puzzling, since such apoptotic processes are thought to be rapid. The unique metabolic characteristics of chondrocytes and the presence of an extensive extracellular matrix may maintain local conditions sufficient to sustain enzyme activities and their substrates long after cell death. Longer-term studies in either an ex vivo perfusion system or even utilizing an animal model would be 

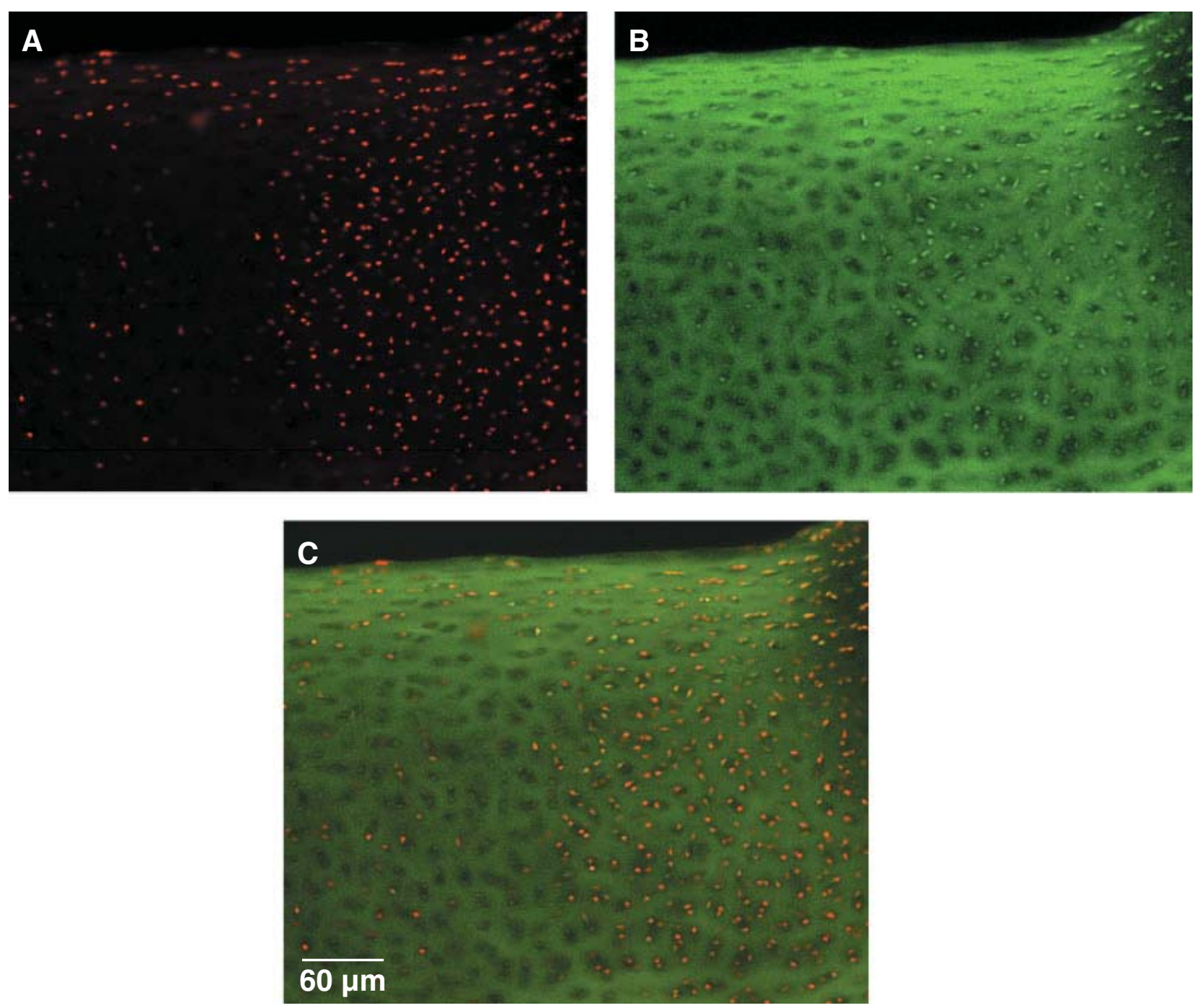

Figure 5. Bovine cartilage $24 \mathrm{~h}$ following holmium:YAG laser exposure $(780 \mathrm{~mJ})$ and incubated with PhiPhilux ${ }^{\mathrm{TM}}$ to indicate caspase-3 activity (green fluorescence) and ethidium homodimer-I to mark dead cells with disrupted cell membranes (red fluorescence). (A) and (B) represent the individual signals of ethidium and PhiPhilux respectively. The overlay of both images (C) indicates that these two markers co-signal in the same cell after holmium:YAG-induced damage

required, as we noted viability problems in some samples cultured beyond 6 days (data not supplied).

The TUNEL method has received a mixed review for its reliability in discriminating DNA fragmentation as a consequence of apoptosis and necrosis [29-33]. Our observations here provide grounds for caution. The specificity of TUNEL may be 'fine tuned' by correct controls, such as the use of growth plate material [8], to distinguish apoptosis-derived nucleic acid degradation. Alternatively, we show in this study that by combining other signposts of apoptosis, such as caspase activity and cell morphology (TEM), one may discriminate between such processes.

Cells with plasma membranes permeable to ethidium homodimer-1 and with fragmented DNA (TUNEL positive) are in the late stages of apoptosis and are not dying from ACD, as is confirmed by positive caspase3 signals. This indicates that DNA fragmentation and the loss of plasma membrane integrity are two processes that commence either simultaneously or within a short time of each other. It would seem that the
TUNEL signals measured by day 1 represent mainly necrotic cells, as disclosed by the TEM images. The progression of the TUNEL signal over time appears to be due to an apoptotic process, because of the detection of active caspase- 3 and the morphology of cells located in the peripheral area of damage. Once more, this highlights the fact that a TUNEL signal alone may be a misleading marker of apoptotic events and thus should be combined with other markers for the delineation of necrosis and apoptosis.

The mitochondrial membrane potential marker, CMXRos, revealed a signal pattern very similar to calcein-AM and may therefore provide an alternative means of assessing cell viability. The resolution of CMXRos (red) combined with PhiPhilux (green) was not clear (background green stain) and thus its use alone, or in combination with ethidium homodimer (red), may be more interesting. This marker of mitochondrial integrity may also be useful to investigate the regulatory role of this organelle in controlling apoptotic events in chondrocytes. 


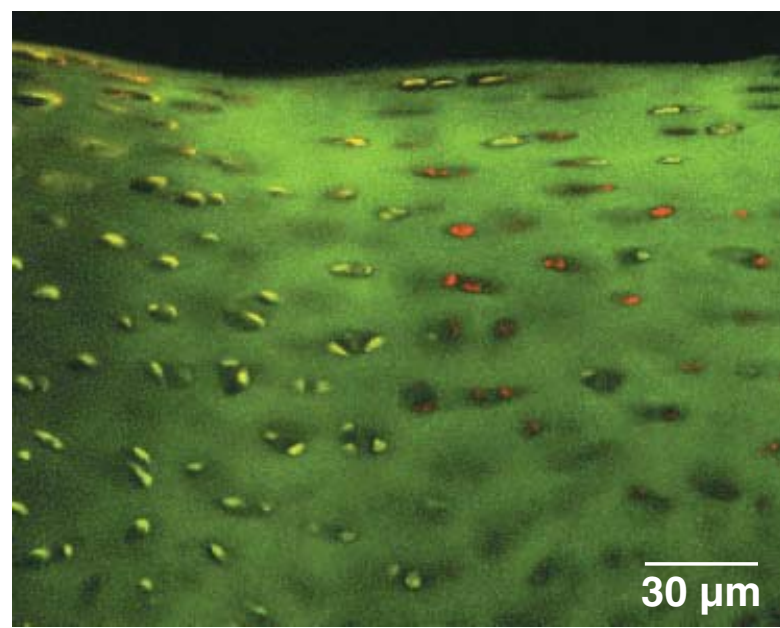

Figure 6. Confocal LSM visualization of caspase-3 activity (PhiPhilux ${ }^{\text {TM }}$ green fluorescence) and mitochondrial membrane potential (CMXRos, red fluorescence) of bovine articular cartilage following holmium:YAG laser exposure. Laser damage, to the left, is shown by a strong caspase- 3 activity and a lack of mitochondrial (red) signal. Adjacent to the damage are red fluorescent cells that do not have active caspase- 3 signals and are thus classified as healthy, i.e. without the loss of mitochondrial membrane potential

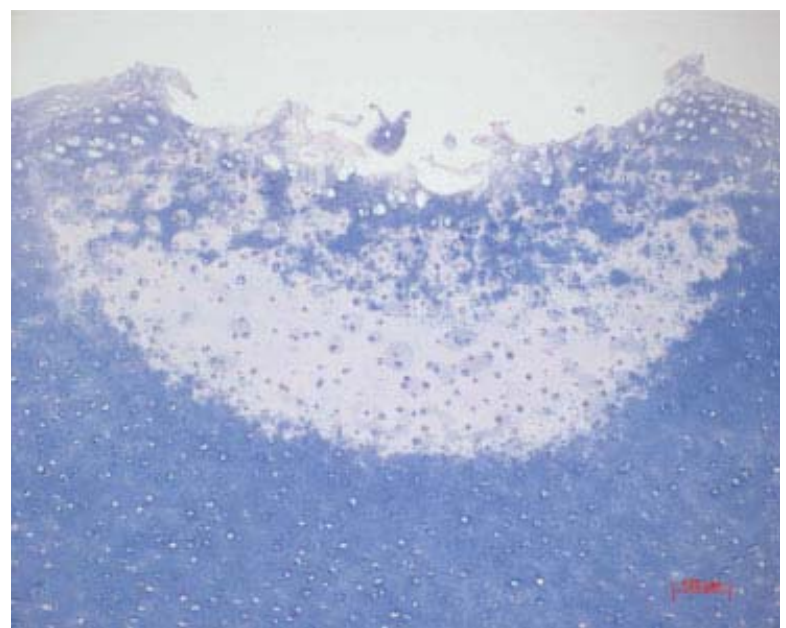

Figure 7. A paraffin-embedded cartilage section stained with alcian blue. The holmium:YAG laser damage is apparent by the reduction in matrix staining. Pyknotic cell morphology is evident outside the area of matrix loss. Evaluation based only on loss of matrix components underestimates laser-induced damage and the assessment of a boundary of pycnotic cellular morphology is not precise
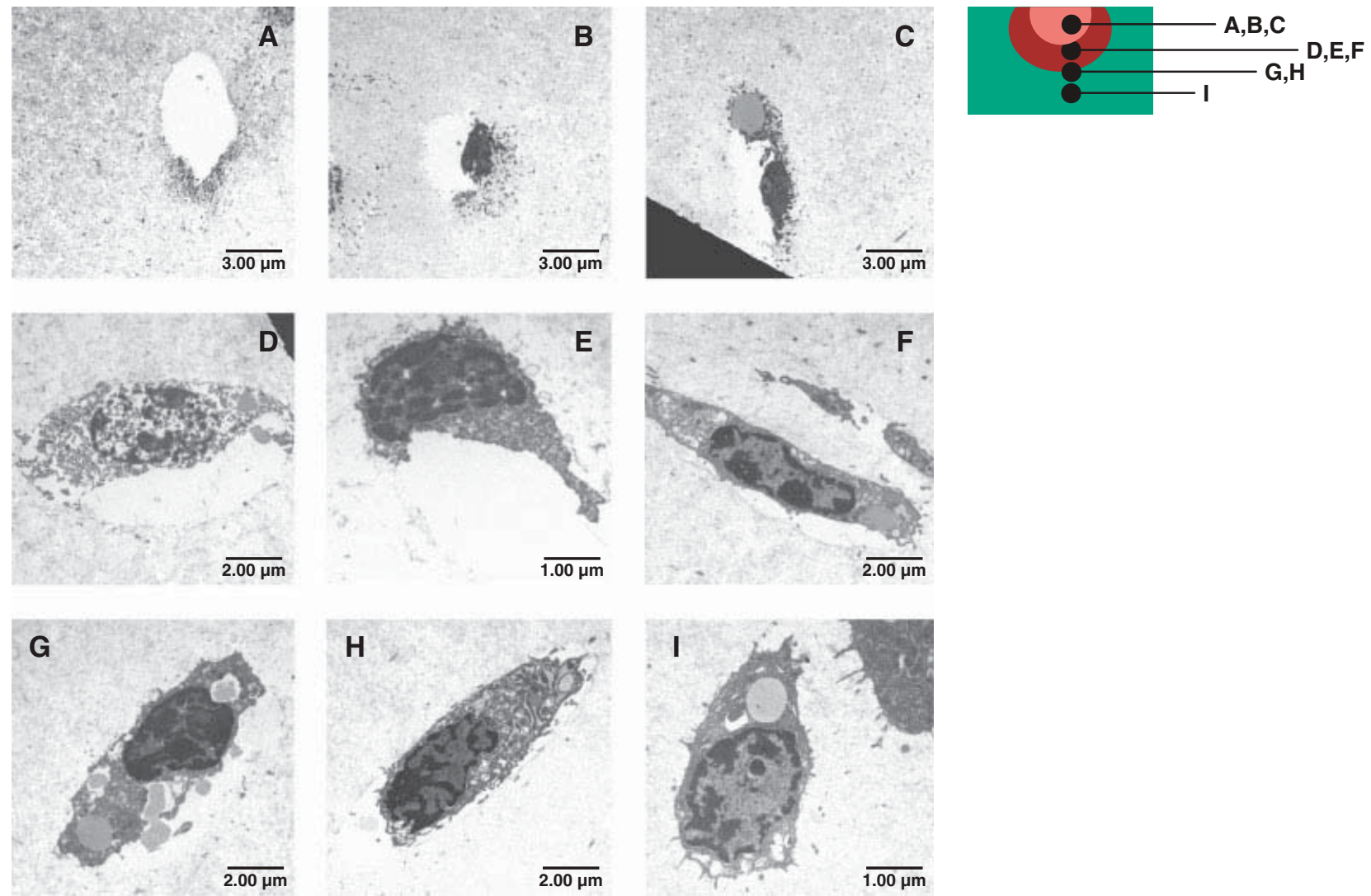

Figure 8. Transmission electron micrographs of chondrocytes in bovine cartilage one day after holmium: YAG laser exposure $(780 \mathrm{~mJ}) .(A),(B)$ and $(C)$ reveal cells (or remnants) that are located closer to the mid-section of laser impact area (zone I, see Figure I). These cells are predominantly vaporized and cellular contents are forced into the immediate surrounding matrix. (D), (E) and $(\mathrm{F})$ are examples of cells located in zone 2a. A mixture of necrosis and perhaps nuclear condensation may suggest apoptosis. 'Classical' cell apoptosis morphology was not apparent in these cells, which gave an active caspase- 3 and ethidium-positive signal. These same cells became TUNEL positive by day 6. $(\mathrm{G})$ and $(\mathrm{H})$ represent cells that are located in zone $2 \mathrm{~b}$, towards the outer edge of the ethidium homodimer boundary. These chondrocytes appear almost morphologically normal, with only slight cellular disorganization. The two chondrocytes in I are located outside the ethidium area and in the calcein-positive region (zone 3) and appear intact 
It has been reported that chondrocytes do not display the morphological features of 'classical' apoptosis as seen in other cell types, but may assume a morphology consistent with 'dark chondrocytes' [34]. Related to this atypical morphology, Kass et al. [35] showed that chromatin condensation during apoptosis requires ATP, giving rise to alterations in cellular morphology, but DNA fragmentation can still occur without this energy substrate. Chondrocytes are reported to be less metabolically active than other cell types [36] and, coupled with the static culture conditions in this study, lower ATP levels may have prevented the development of typical apoptotic morphology, while allowing normal processes to continue to fragment DNA, as indicated by the progression of the TUNEL signal. The unusual characteristics of chondrocytes, also illustrated by the unexpected combined co-signal of caspase-3 and ethidium, suggest that their apoptotic processes may differ from those observed in other cell types.

The model described here may be useful in the assessment and development of a variety of other viability and apoptosis markers. Laser injury was chosen since it is precise and reproducible; it produces necrotic and apoptotic damage in ex vivo cartilage and thus permits studies of the balance between these two events. We envisage the routine use of the reported markers in the assessment of orthopaedic instrumentation and in the evaluation of tissue-engineered constructs. Moreover, this or similar models may also aid in the study of apoptosis and tissue viability in toxicology, experimental pathology and immunobiology, for instance, in studies of cartilage diseases such as osteoarthritis and rheumatoid arthritis, where the involvement of apoptosis is contentious [11,37,38]. The easy procurement of a living solid tissue, its excellent cutting properties in the native state for sampling, its good optical properties for LSM analysis and the ease of culture follow-up invite such experiments. Procedures could thus be optimized before testing in expensive animal experiments.

\section{Acknowledgements}

We are grateful for the technical assistance of Mrs Verena Winkelmann (TEM and histology preparations), Dr Beat Ott (holmium: YAG laser treatments) and Dr Christoph Weiler. This work was supported by the Swiss Commission for Technology and Innovation (CTI: 4716.1) and the Swiss National Funds (SNF: 4046-58623).

\section{References}

1. Thompson CB. Apoptosis in the pathogenesis and treatment of disease. Science 1995; 267: 1456-1462.

2. Nagata S. Apoptosis by death factor. Cell 1997; 88: 355-365.

3. Blankenberg FG, Tail JF, Strauss HW. Apoptotic cell death: its implications for imaging in the next millennium. Eur J Nucl Med 2000; 27(3): 359-367.

4. Errington RJ, Fricker MD, Wood JL, Hall AC, White NS. Fourdimensional imaging of living chondrocytes in cartilage using confocal microscopy: a pragmatic approach. Am J Physiol 1997; 272(3 Pt 1): C1040-C1051.
5. Lu Y, Edwards RB 3rd, Kalscheur VL, Nho S, Cole BJ, Markel MD. Effect of bipolar radiofrequency energy on human articular cartilage: comparison of confocal laser microscopy and light microscopy. Arthroscopy 2001; 17(2): 117-123.

6. Mainil-Varlet P, Monin D, Weiler C, et al. Quantification of laserinduced cartilage injury by confocal microscopy in an ex vivo model. J Bone Joint Surg Am 2001; 83-A(4): 566-571.

7. Gibson G. Active role of chondrocyte apoptosis in endochondral ossification. Microsc Res Tech 1998; 43(2): 191-204.

8. Aigner T, Hemmel M, Neureiter D, et al. Apoptotic cell death is not a widespread phenomenon in normal aging and osteoarthritis human articular knee cartilage: a study of proliferation, programmed cell death (apoptosis), and viability of chondrocytes in normal and osteoarthritic human knee cartilage. Arthritis Rheum 2001; 44(6): 1304-1312.

9. James IE, Kumar S, Barnes MR, et al. FrzB-2: a human secreted frizzled-related protein with a potential role in chondrocyte apoptosis. Osteoarthritis and Cartilage 2000; 8(6): 452-463.

10. Takahashi K, Hashimoto S, Kubo T, Hirasawa Y, Lotz M, Amiel D. Effect of hyaluronan on chondrocyte apoptosis and nitric oxide production in experimentally induced osteoarthritis. J Rheumatol 2000; 27(7): 1713-1720.

11. Yatsugi N, Tsukazaki T, Osaki M, Koji T, Yamashita S, Shindo H. Apoptosis of articular chondrocytes in rheumatoid arthritis and osteoarthritis: correlation of apoptosis with degree of cartilage destruction and expression of apoptosis-related proteins of p53 and c-myc. J Orthop Sci 2000; 5(2): 150-156.

12. Loening AM, James IE, Levenston ME, et al. Injurious mechanical compression of bovine articular cartilage induces chondrocyte apoptosis. Arch Biochem Biophys 2000; 381(2): 205-212.

13. Chen CT, Burton-Wurster N, Borden C, Hueffer K, Bloom SE, Lust G. Chondrocyte necrosis and apoptosis in impact damaged articular cartilage. J Orthop Res 2001; 19(4): 703-711.

14. Kim HA, Suh DI, Song YW. Relationship between chondrocyte apoptosis and matrix depletion in human articular cartilage. $J$ Rheumatol 2001; 28(9): 2038-2045.

15. Mansfield K, Rajpurohit R, Shapiro IM. Extracellular phosphate ions cause apoptosis of terminally differentiated epiphyseal chondrocytes. J Cell Physiol 1999; 179(3): 276-286.

16. Malicev E, Woyniak G, Knezevic M, Radosavljevic D, Jeras M. Vitamin $\mathrm{C}$ induced apoptosis in human articular chondrocytes. Pflugers Arch 2000; 440(5 Suppl): R46-48.

17. Renvoize C, Biola A, Pallardy M, Breard J. Apoptosis: identification of dying cells. Cell Biol Toxicol 1998; 14(2): 111-120.

18. Majno G, Joris I. Apoptosis, oncosis, and necrosis: an overview of cell death. Am J Pathol 1995; 146(1): 3-15.

19. Kerr JF, Wyllie AH, Currie AR. Apoptosis: a basic biological phenomenon with wide-ranging implications in tissue kinetics. $\mathrm{Br}$ J Cancer 1972; 26(4): 239-257.

20. Kroemer G, Dallaporta B, Resche-Rigon M. The mitochondrial death/life regulator in apoptosis and necrosis. Annu Rev Physiol 1998; 60: 619-642.

21. Kroemer G. Mitochondrial control of apoptosis: an overview. Biochem Soc Symp 1999; 66: 1-15.

22. Desagher S, Martinou JC. Mitochondria as the central control point of apoptosis. Trends Cell Biol 2000; 10(9): 369-377.

23. Budihardjo I, Oliver H, Lutter M, Luo X, Wang X. Biochemical pathways of caspase activation during apoptosis. Annu Rev Cell Dev Biol 1999; 15: 269-290.

24. Mainil-Varlet P, Monin D, Weiler C, et al. Quantification of laserinduced cartilage injury by confocal microscopy in an ex vivo model. J Bone Joint Surg Am 2001; 83-A(4): 566-571.

25. Namura $S$, Zhu J, Fink K, et al. Activation and cleavage of caspase-3 in apoptosis induced by experimental cerebral ischemia. J Neurosci 1998; 18(10): 3659-3668.

26. Puka-Sundvall M, Wallin C, Gilland E, et al. Impairment of mitochondrial respiration after cerebral hypoxia-ischemia in immature rats: relationship to activation of caspase- 3 and neuronal injury. Brain Res Dev Brain Res 2000; 125(1-2): 43-50.

27. Yamazaki Y, Tsuruga M, Zhou D, et al. Cytoskeletal disruption accelerates caspase-3 activation and alters the intracellular 
membrane reorganization in DNA damage-induced apoptosis. Exp Cell Res 2000; 259(1): 64-78.

28. Liu X, Zou H, Slaughter C, Wang X. DFF, a heterodimeric protein that functions downstream of caspase-3 to trigger DNA fragmentation during apoptosis. Cell 1997; 89(2): 175-184.

29. Charriaut-Marlangue C, Ben-Ari Y. A cautionary note on the use of the TUNEL stain to determine apoptosis. Neuroreport 1995; 7 : $61-64$.

30. Grasl-Kraupp B, Ruttkay-Nedecky B, Koudelka H, Bukowska K, Bursch W, Schulte-Hermann R. In situ detection of fragmented DNA (TUNEL assay) fails to discriminate among apoptosis, necrosis, and autolytic cell death: a cautionary note. Hepatology 1995; 21(5): 1465-1468.

31. Nakamura M, Yagi H, Kayaba S, et al. Most thymocytes die in the absence of DNA fragmentation. Arch Histol Cytol 1995; 58(2): 249-256.

32. Cervos-Navarro J, Schubert TE. Pitfalls in the evaluation of apoptosis using TUNEL. Brain Pathol 1996; 6(3): 347-348.
33. de Torres C, Munell F, Ferrer I, Reventos J, Macaya A. Identification of necrotic cell death by the TUNEL assay in the hypoxic-ischemic neonatal rat brain. Neurosci Lett 1997; 230(1): $1-4$.

34. Roach HI, Clarke MP. Physiological cell death of chondrocytes in vivo is not confined to apoptosis. J Bone Joint Surg (Br) 2000; 82-B(4): 601-613.

35. Kass GE, Eriksson JE, Weis M, Orrenius S, Chow SC. Chromatin condensation during apoptosis requires ATP. Biochem J 1996; 318(Pt 3): 749-752.

36. Stockwell RA. Morphology of cartilage. In Methods in Cartilage Research. Maroudas A, Kuettner K (eds). Academic Press: London, 1990; 62.

37. Lotz M, Hashimoto S, Kuhn K. Mechanisms of chondrocyte apoptosis. Osteoarthritis Cartilage 1999; 7(4): 389-391.

38. Lozoya KA, Flores JB. A novel rat osteoarthrosis model to assess apoptosis and matrix degradation. Pathol Res Pract 2000; 196(11): $729-745$. 